\title{
Limiting acceptance angle to maximize efficiency in solar cells
}

\author{
Emily D. Kosten ${ }^{a}$ and Harry A. Atwater ${ }^{a, b}$ \\ ${ }^{a}$ Thomas J. Watson Laboratories of Applied Physics, California Institute of Technology, \\ Pasadena, California 91125, USA; \\ ${ }^{b}$ Kavli Nanoscience Institute, California Institute of Technology, Pasadena, California 91125, \\ USA
}

\section{ABStract}

Within a detailed balance formalism, the open circuit voltage of a solar cell can be found by taking the band gap energy and accounting for the losses associated with various sources of entropy increase. Often, the largest of these energy losses is due to the entropy associated with spontaneous emission. This entropy increase occurs because non-concentrating solar cells generally emit into $2 \pi$ steradian, while the solid angle subtended by the sun is only $6.85 \times 10^{-5}$ steradian. Thus, for direct normal irradiance, non-concentrating solar cells with emission and acceptance angle limited to a narrow range around the sun could see significant enhancements in open circuit voltage and efficiency. With the high degree of light trapping we expect given the narrow acceptance angle and the ray optics brightness theorem, the optimal cell thickness will result in a discrete modal structure for most materials. Thus, limiting the acceptance and emission angle can be thought of as coupling to only a subset of radiating modes, or, alternatively, as altering the modal structure such that some radiating modes become bound modes. We have shown the correspondence between the ray optics picture and the modal picture, by deriving the ray optics results for light trapping under angular restrictions using a modal formulation. Using this modal formulation we can predict the light trapping and efficiencies for various thin structures under angular restriction. We will discuss these predicted efficiencies and various options for implementing broadband and angle-specific couplers.

\section{INTRODUCTION}

In a traditional flat-plate solar cell under direct sunlight, light is received from only the direction of the sun, but it is emitted in all directions. The emission of light in all directions represents an increase in photon entropy within the system. As with any other entropy increase, the photon entropy increase has a detrimental impact on cell efficiency. More specifically, the open circuit voltage of a solar cell is approximately the semiconductor band gap with the various entropy increase terms subtracted. Thus, increasing entropy corresponds to reduced open circuit voltage. Often, the largest of these entropy increase terms is the photon entropy increase due to isotropic emission under direct sunlight, which corresponds to a nearly $300 \mathrm{mV}$ drop in the open circuit voltage, as will be explained later. Thus, if an ideal cell has its emission angle limited to the disk of the sun, the open circuit voltage approaches the bandgap of the semiconductor solar cell material and the detailed balance solar cell efficiency is $41 \%$ for a single junction. ${ }^{1}$

The most common approach to addressing photon entropy is a concentrator system. With a concentrator light is emitted from the small area of the solar cell with wide angular spread, and reflections in the concentrator array it over a larger area with a narrower angular spread. In essence, a concentrator trades off entropy in the spatial distribution of the light for entropy in the angular distribution of the light. Because of their reduction of the photon entropy term, concentrator systems see efficiency increases, due to increases in open circuit voltage, relative to flat plate cells for low concentration ratios. However, concentrator systems cannot take full advantage of reducing the photon entropy, because maximum reduction of emission angle occurs at maximum concentration. At these high concentrations, losses due to heating and series resistance with increasing short circuit current offset the improved photon entropy term, overall efficiency is reduced. ${ }^{2}$ Since concentrator systems cannot take full advantage of the photon entropy term, we consider a non-concentrating system with limited emission angle. With this system, we can reduce the emission angle to the solar disk without increased heating or series resistance, as short circuit current density is relatively constant.

\footnotetext{
Nonimaging Optics: Efficient Design for Illumination and Solar Concentration VIII, edited by Roland Winston, Jeffrey M. Gordon, Proc. of SPIE Vol. 8124, 81240F (C) 2011 SPIE · CCC code: 0277-786X/11/\$18 - doi: 10.1117/12.894226
}

Proc. of SPIE Vol. $812481240 \mathrm{~F}-1$ 
Mechanistically, addressing photon entropy has two distinct advantages. First, there is photon recycling, where it is more difficult for photons generated by radiative recombination to leave the cell. ${ }^{3}$ Since in an ideal cell this is the only source of dark current, this effect is very significant where non-radiatve recombination is minimal. Second, there is improved light trapping in an ergodic cell, as it is more difficult for unabsorbed photons to leave the cell. ${ }^{4,5}$ While both of these effects have been examined separately in previous work, this work considers a system with an ergodic cell with little non-radiative recombination, so both effects are seen, giving substantial efficiency increases for realistic materials.

Here, we develop a detailed balance model that accounts for limited emission angle for both an ideal and a more realistic cell, and allows us derive the photon entropy term. This allows us to explore design considerations such as the effect of cell thickness, and the tradeoffs between moderate and strict angular restriction. Inherent in this analysis is the consideration of both photon recycling and light trapping.

\section{DETAILED BALANCE MODEL}

\subsection{Steady state photon flux}

We begin with a detailed balance model, as developed by Shockley and Queisser, and elaborated by later workers. ${ }^{3,6-8}$ We assume a cell with no non-radiative recombination. and that each absorbed photon above the bandgap forms one electron-hole pair. Then, at steady state, the flux of photons into the cell from the suns must equal the flux of photons out of the cell from blackbody radiation.

$$
\int_{0}^{\infty} S(E) a(E) d E=\int_{\Omega_{c}} \int_{0}^{\infty} a(E) \frac{2}{h^{3} c^{2}} \frac{E^{2}}{e^{\left(E-q V_{o c}\right) / k T}-1} d E \cos (\theta) d \Omega
$$

Above, $S(E)$ is the AM 1.5 solar spectrum, and $a(E)$ is the absorptivity of the cell, so the left hand side is the absorbed solar photon flux. $\Omega_{c}$ gives the solid angle of emission from the solar cell, and the right hand side gives the emission of the solar cell, which is reduced as $\Omega_{c}$ decreases. To find the current at a given voltage, $V_{o c}$ is replaced by that voltage, and the right hand side is subtracted from the left hand side. Then, the power can be found.

\subsection{Photon recycling and open circuit voltage}

To better understand photon recycling, we assume for illustrative purposes that $E-q V_{o c}>>T$ for all energies and solve for the open circuit voltage.*

$$
q V_{o c} \approx k T \ln \frac{\int_{E_{g}}^{\infty} S(E) a(E) d E}{\int_{\Omega_{c}} \int_{0}^{\infty} a(E) \frac{2}{h^{3} c^{2}} E^{2} e^{-E / k T} d E \cos (\theta) d \Omega} \approx k T \ln \frac{J_{s c}}{J_{0}}
$$

As the solid angle of emission, $\Omega_{c}$, is decreased, the dark current, $J_{0}$, is reduced. This is the voltage increase from photon recycling. The solid angle of emisssion is defined by a half-angle of emisssion $\theta$, which can vary between $.267^{\circ}$, the half angle of the sun, and $90^{\circ}$. As the solid angle of emisssion varies between these two limits, the open circuit voltage is reduced by approximately $280 \mathrm{mV}$, giving the result mentioned in the introduction. Because a concentrator system addresses the same entropy term, this equation should give the same open circuit voltage result for under maximum concentration. In a concentrator system, rather than reducing $J_{0}$, the short circuit current is increased by the same factor at maximum concentration. This gives the same potential voltage and efficiency gains for a given angular restriction. However, as previously discussed, the increased short circuit current also causes increased heating and series resistance, so a concentrator cell will not realize the full potential voltage gain. By not concentrating, the open circuit voltage increases without a short circuit current increase because the dark current, rather than the short circuit current, changes in the above expression. Thus, the full potential gains from reducing the photon entropy can be realized.

\footnotetext{
${ }^{*}$ This assumption will not be true in all the cases we consider, and we do not assume it for our calculations.
} 


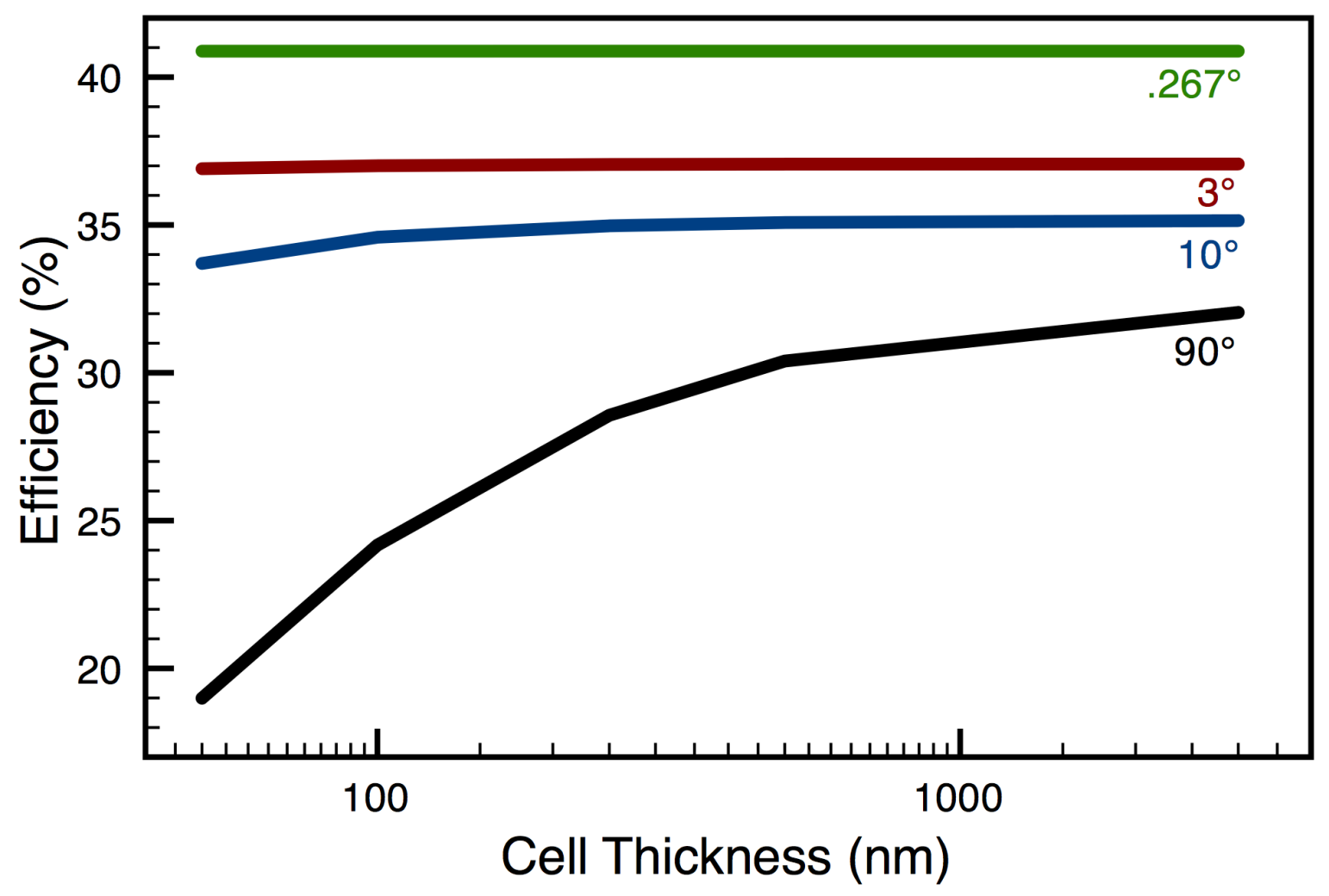

Figure 1. Detailed balance efficiencies as a function of cell thickness for GaAs solar cells with thick silicon nitride cladding, assuming no non-radiative recombination. The labels on each line give the emission angle, with $.267^{\circ}$ corresponding to the solar disk, and $90^{\circ}$ corresponding to no emission angle restriction. The minimum cell thickness is $50 \mathrm{~nm}$ and maximum is 3 microns. As the cell thickness increases more of the light is absorbed on a single pass, so the enhanced light trapping with decreased angle of emission provides less of an advantage. The photon recycling advantage is independent of thickness in this case, so the advantage

\subsection{Evaluating absorptivity}

Next, we must evaluate the absorptivity of the solar cell as a function of photon energy, solar cell thickness, and solar cell refractive index. In the ray optics limit, the expression for $a(E)$ is well-known:

$$
a(E)=\frac{\alpha(E)}{\alpha(E)+\sin ^{2}(\theta) / 4 n^{2} W}
$$

where $\alpha$ is the absorption length in the semiconductor, $W$ is the cell thickness, and $n$ is the relative index of refraction of the cell material. ${ }^{4,5}$ However, this expression will not be valid for thin cells, where the material can no longer be treated as bulk. Since increased absorptivity with limited emission angle should allow for thinner cells, we use a model to treat the absorptivity that accounts for the modal structure in thin cells, assuming the cell is clad by thick layers of silicon nitride. ${ }^{9}$ Thus, we can accurately evaluate the absorptivity in very thin cells.

\subsection{Results for an idealized cell}

Figure 1 gives the results found using the absorptivity model from above, as a function of cell thickness for a GaAs solar cell with no non-radiative recombination. We find efficiencies above $40 \%$ are possible in this idealized cell with emission angle limited to the solar disk. As the emission angle is widened, performance decreases, as we expect. There is a fairly significant difference between the result for $3^{\circ}$ and for $.267^{\circ}$, corresponding to the solar 


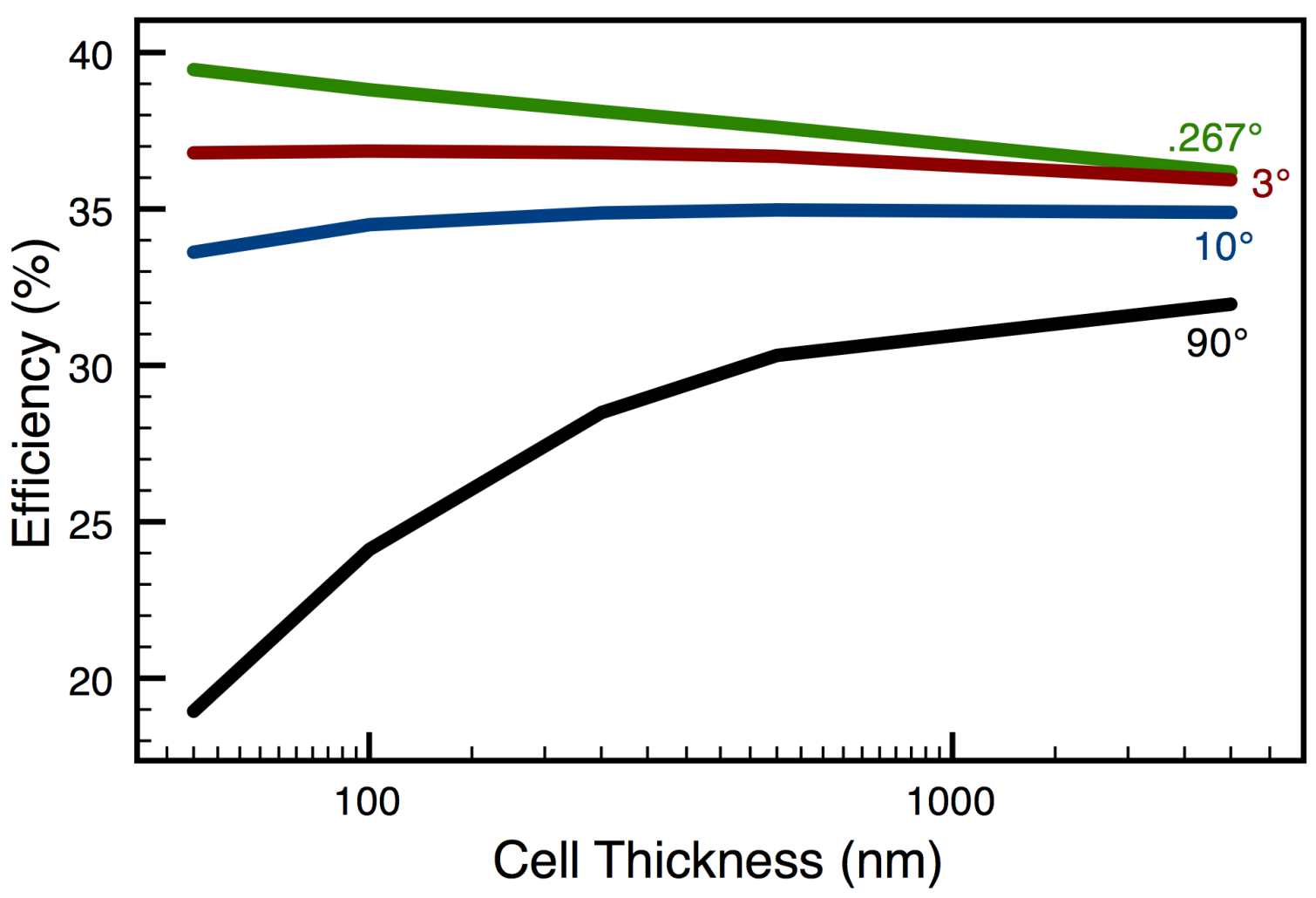

Figure 2. Detailed balance efficiencies as a function of cell thickness for GaAs solar cells with thick silicon nitride cladding, and $90 \%$ internal fluorescence yield. The labels on each line give the emission angle, with $.267^{\circ}$ corresponding to the solar disk, and $90^{\circ}$ corresponding to no emission angle restriction. The performance is reduced relative to the idealized case, and the behavior with thickness is different.

disk. This suggests that very narrow angular restriction is preferable, though difficulties in tracking must be considered. In addition, when limiting emission and acceptance angle to the solar disk, the effects of circumsolar radiation must also be considered.

In the case where the emission angle is not limited, marked $90^{\circ}$ on the plot, performance falls up rapidly with decreasing thickness, as we would expect. However, for a cell with emission angle limited to the solar disk, or $.267^{\circ}$, there is little decrease in efficiency as the cell is thinned because light trapping is so strongly enhanced as the emission angle is limited. Thus, for maximal emission angle restriction, we can go down to a $50 \mathrm{~nm}$ thick cell, using less than $2 \%$ of the GaAs in a more traditional 3 micron thick cell, without sacrificing performance. This represents a very substantial materials savings, corresponding to the materials savings of a $60 \mathrm{x}$ concentration system.

While the performance increase is greatest for thin cells with limited emission angle, as shown in figure 1, there is still a substantial improvement even for a more traditional thick cell with limited emission angle. This is purely due to the increase in open circuit voltage discussed previously, since at this thickness light trapping is sufficient for nearly all the light above the bandgap to be absorbed. Thus, the short circuit currents are very similar regardless of emission angle limitation. This is in contrast to thinner cells, where the short circuit current is also greater when emission angle is limited, because increased light trapping allows nearly all the light to be absorbed. 


\subsection{Effect of non-idealities}

In any realistic material, however, there will be some non-radiative recombination. Thus, the results above are not achievable in any real material. We therefore include the effects of non-radiative recombination in our model. We account for the non-radiative recombination by including it in the dark current, and relate it to the internal fluorescence yield. ${ }^{10,11}$ Incorporating the non-radiative recombination in this way allows us to treat material quality as an adjustable parameter. Thus, for a material such as GaAs, which suffers from relatively little non-radiative recombination in good quality material, we choose a large value for internal fluorescence yield. However, in the case of silicon, a smaller value for internal fluorescence yield would be more realistic, owing to Auger recombination.

For this calculation we set internal fluorescence yield to $90 \%$, a very reasonable value for GaAs. The results are shown in figure 2. As before, the advantages of limiting acceptance angle are greatest for thinner cells, and fall off, but are still present for thicker cells. This can be explained in the context of light trapping and photon recycling, and is analogous to the non-ideal case. However, in comparison to figure 1, we see that, the efficiencies are considerably lower. Furthermore, the advantage of very strict emission angle limitation is diminished somewhat, as the amount non-radiative recombination increases with decreased emission angle.

When non-radiative recombination is included, the efficiency actually decreases as the cells get thicker for cells with limited emission angle. This is because in a thicker cell it is more difficult for light resulting from radiative recombination to escape the cell, so there is more non-radiative recombination. Enhanced light trapping maintains the absorptivity in a thinner cell, so the non-radiative recombination effect is dominant. In contrast, this efficiency decrease does not occur for thicker cells without a limited emission angle because the increased non-radiative recombination effect is outweighed by decreased absorptivity. Thus, thinner cells are actually preferable for very strict angular restriction, which would also give significant materials savings.

While accounting for more realistic materials behavior in this detailed balance model, reduces the predicted efficiency increases somewhat, they are still quite impressive. Furthermore, including non-radiative recombination reveals that thinner solar cells lead to significantly enhanced performance under conditions where the emission angle is limited. In addition, the benefits to very strict angle restriction are greatest for the thinnest cells because the increases in non-radiative recombination with reduced angle are mitigated as the cell is thinned. Thus, to maximize performance in a GaAs cell with this approach, the acceptance angle should be strictly limited, and the cell should be very thin.

\section{CONCLUSIONS AND OUTLOOK}

Using a detailed balance model, we have considered the efficiency implications of limiting the emission angle of an ergodic, ideal GaAs cell with varying thickness. Initially neglecting non-radiative recombination, we find that significant benefits are possible in an idealized system, and that these benefits increase with decreased emission angle. Furthermore, in this idealized system, we find very strict emission angle restriction gives significant advantages over only moderate angular restriction. Finally, the improvement with limited emission in an idealized system is greatest for thin cells because the performance of a non-restricted cell degrades, owing to decreased absorptivity, while an angle restricted cell maintains its absorptivity via enhanced light trapping. This enhanced light trapping also opens up the possibility for significant materials savings via thinner cells.

We then considered a non-ideal cell by accounting for non-radiative recombination. For a GaAs cell, with a reasonable $90 \%$ internal fluorescence yield, cells with limited emission angle continued to show significantly higher efficiencies than a cell without limited emission angle. However, thinner cells with strictly limited emission actually outperformed their thicker counterparts, owing to the reduced effects of non-radiative recombination in these cells in combination with enhanced light trapping due to the strictly limited emission. Thus, in contrast to the idealized case, where there is little difference between a thin and a thick cell under strict angular restriction, a thinner cell is definitely preferable in this more realistic case. Additionally, for this case the advantages of strictly limited emission as opposed to moderately limited emission are minimal for thick cells, and reduced from the idealized case for thin cells. Optimally, then a very thin cell with strictly limited emission would be used. However, if a thicker cell were to be used, it is not necessary to limit the emission angle as strictly.

Proc. of SPIE Vol. $812481240 F-5$ 
Here, we have explored via a detailed balance model design considerations for an ergodic GaAs solar cell with limited emission angle. We have considered both an idealized and a non-ideal case, and have found that the benefits to limiting emission angle are robust to the limitations of real materials, despite being reduced relative to the idea case. In addition, we find that the more realistic case shows substantially different behavior which would influence the design choices for such a cell.

\section{ACKNOWLEDGMENTS}

The authors thank O. Miller for his advice on handling non-radiative recombination and R. Briggs for his advice on mode structure calculations. D. Callahan, M. Sheldon, and A. Tamboli also provided very useful discussions. This work was supported by the Light-Matter Interactions Energy Frontier Research Center, an EFRC program of the office of science, United States Department of Energy under grant DE-SC0001293.

\section{REFERENCES}

1. Araújo, G. and Martí, A., "Absolute limiting efficiencies for photovoltaic energy conversion," Solar Energy Mater. and Solar Cells 33, 213-240 (1994).

2. Green, M., [Solar Cells: Operating Principles, Technology, and System Applications], Prentice-Hall (1982).

3. Martí, A., Balenzategui, J., and Reyna, R., "Photon recycling and Shockely's diode equation," J. Appl. Phys. 82(8), 4067-4075 (1997).

4. Campbell, P. and Green, M., "The limiting efficiency of silicon solar cells under concentrated sunlight," IEEE Trans. on Elec. Dev. ED-33, 234-239 (1986).

5. Yablonovitch, E., "Statistical ray optics," J. Opt. Soc. Am. 72(7) (1982).

6. Shockley, W. and Queisser, H., "Detailed balance limit of efficiency of p-n junction solar cells," J. Appl. Phys. 32(3), 510-519 (1961).

7. Tiedje, T., Yablonovitch, E., Cody, G., and Brooks, B., "Limiting efficiency of silicon solar cells," IEEE Trans. on Elec. Dev. ED-31, 711-716 (1984).

8. Araújo, G. and Martí, A., "Limiting efficiencies of GaAs solar cells," IEEE Trans. Elec. Dev. 37(5), 14021405 (1990).

9. Stuart, H. and Hall, D., "Thermodynamic limit to light trapping in thin planar structures," J. Opt. Soc. Am. A. 14(11), 3001-3008 (1997).

10. Ross, R., "Some thermodynamics of photochemical systems," J. Chem. Phys. 46(12), 4590-4593 (1967).

11. Schnitzer, I., Yablonovitch, E., Caneau, C., and Gmitter, T., "Ultrahigh spontaneous emission quantum efficiency, $99.7 \%$ internally and $72 \%$ eternally, from AlGaAs/GaAs/AlGaAs double heterostructures," Appl. Phys. Lett. 62(2), 131-133 (1992). 